\title{
Welche Berufsgruppen sind am meisten gefährdet?
}

\author{
Wie viel Sonne bekommt ein Kanalbauer ab und wie viel ein Landwirt? Um die Frage nach der indivi- \\ duellen UV-Exposition und damit dem Hautkrebsrisiko einzelner Berufsgruppen beantworten zu \\ können, führt das Institut für Arbeitsschutz (IFA) der Deutschen Gesetzlichen Unfallversicherung derzeit \\ die GENESIS-UV-Studie durch. Studienleiter Dr. Marc Wittlich schildert erste Ergebnisse.
}



MMW: Welches sind die bemerkenswertesten Ergebnisse Ihrer Studie?

Wittlich: Mich hat die unglaubliche Bandbreite der Ergebnisse überrascht. Zwischen 40 und 600 SED $^{*}$ ist alles vertreten. Dabei gehen nicht etwa nur die Bauberufe mit relativ hoher Exposition einher. Vielmehr gibt es auch im Baubereich Tätigkeiten, bei denen die Beschäftigten überraschend wenig exponiert sind. Kranfahrer in Hochkränen etwa bekommen nur ungefähr 40 SED ab. Das zeigt, dass man im Kran durch die Scheiben sehr gut gegen Sonne geschützt ist. Baggerfahrer sind dagegen relativ stark exponiert, weil sie häufig mit offener Tür arbeiten und von der Maschine absteigen. Bau ist eben nicht gleich Bau.

MMW: Welche Berufsgruppe ist am stärksten exponiert?

Wittlich: Das sind die Kanalbauer. Diese befinden sich, wenn sie z. B. ein Neubaugebiet erschließen, auf freier Ebene, wo sie der vollen Sonne ausgesetzt sind. Dagegen sind Beschäftigte in der Landwirtschaft im Schnitt weniger exponiert als vermutet. Beim Bestellen großer Felder geht der Trend deutlich zu klimatisierten Traktoren und Mähdreschern. Bei

Abb. 1 Berufe mit der höchsten UV-Strahlenbelastung

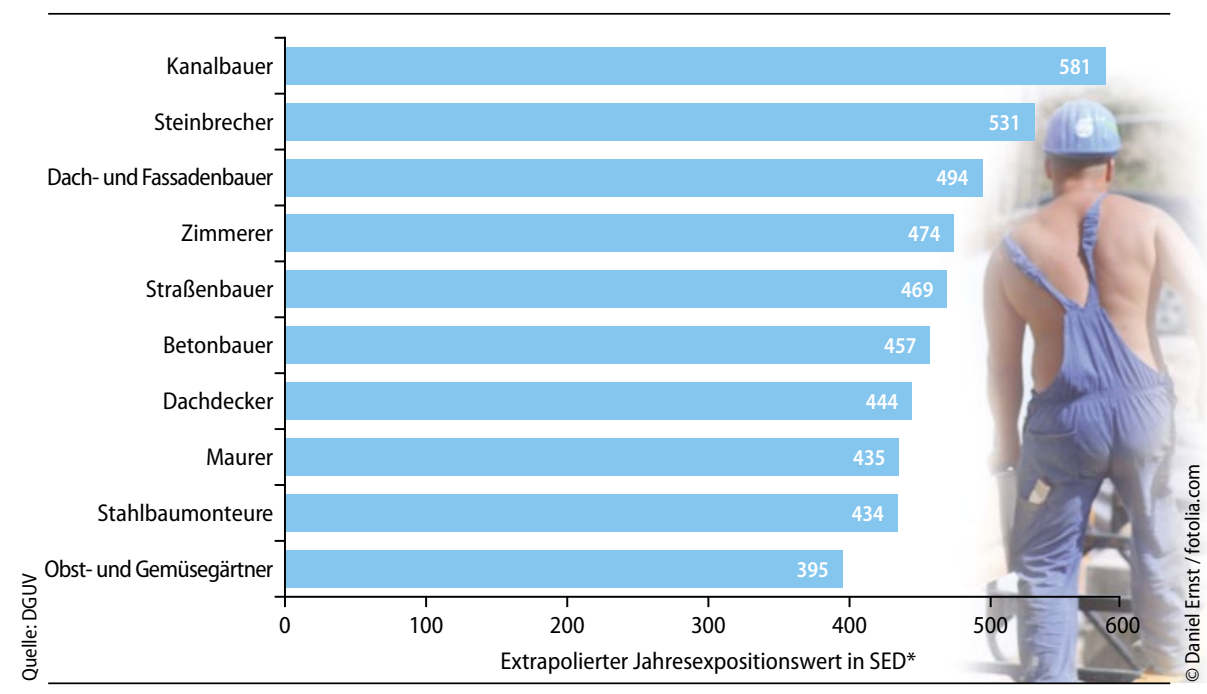

denen muss man nicht mehr das Fenster offen halten, um Luft zu bekommen.

\section{MMW: Was lässt sich im Hinblick auf Präventionsmaßnahmen aus den Daten herauslesen?}

Wittlich: Unser Ziel ist es, maßgeschneiderte Präventionsmaßnahmen zu entwickeln. Beim beruflichen Sonnenschutz stehen an erster Stelle technische Maßnahmen, z.B. das Aufstellen von Sonnensegeln. Danach kommen organisatorische Maßnahmen wie das Verlegen der Arbeitszeit auf Tageszeiten mit geringerer Exposition. Unter den persönlichen Maßnahmen ist der textile Schutz am wichtigsten. Hier reicht ein eng gewebtes Baumwoll-Shirt. Grob lässt sich sagen: Kleidung schützt, wenn man nicht hindurchsehen kann. Das Eincremen mit Sonnencreme ist dagegen weder Plan A noch Plan B, sondern Plan Z. Interview: Dr. Elke Oberhofer

*Standard-Erythem-Dosis: die Menge an UV-Strahlung, die bei einem hellhäutigen rothaarigen Menschen Sonnenbrand auslösen kann.

\section{Das Design der GENESIS-UV-Studie}

In der GENESIS-UV-Studie werden seit 2014 jährlich 300 Probanden über die Unfallversicherungsträger rekrutiert. Die Sonnenexposition wird über ein Messsystem erfasst, das aus einem Dosimeter und einem Tablet-Computer, besteht. Das Dosimeter wird zu Beginn jedes Arbeitstags an der Kleidung befestigt. Am Ende jeder Woche werden die Daten mithilfe einer verschlüsselten Mobilfunkverbindung an das IFA übermittelt. 